\title{
Japanese Community Pharmacists' Barriers to Conducting or Participating in Practice Research
}

\author{
Yasuhiro Sawada',2, Rieko Takehira1, Shigeo Yamamura ${ }^{*}$ \\ ${ }^{1}$ Faculty of Pharmaceutical Sciences, Josai International University, Chiba, Japan \\ ${ }^{2}$ Welcia Yakkyoku Co., Ltd., Tokyo, Japan \\ Email: ${ }^{*}$ s_yama@jiu.ac.jp
}

Received 13 August 2015; accepted 7 September 2015; published 10 September 2015

Copyright (C) 2015 by authors and Scientific Research Publishing Inc.

This work is licensed under the Creative Commons Attribution International License (CC BY).

http://creativecommons.org/licenses/by/4.0/

(c) (i) Open Access

\begin{abstract}
Objectives: This study identified barriers to Japanese community pharmacists' active conduct or participation in practice research. Methods: Community pharmacists $(n=478)$ who gave presentations at three major pharmacy-related conferences in 2012 and 2013 were questioned about their difficulties of giving presentations, support for better presentations, and barriers to conducting pharmacy practice research in their practical setting. A questionnaire was mailed to and returned by the pharmacists directly. Results: We obtained 230 responses (47.9\%). Presentation difficulties included pharmacists' time constraints and lack of experience organizing the report's results or discussion. Many thought statistical analysis support was necessary. The barriers were in sufficient time, community pharmacies' lack of research supervisors, and other community pharmacists' lack of understanding practice research's importance. These were comparable to pharmacists' barriers in other countries, except for money and funds. Conclusions: Japanese community pharmacists should clarify that practice research in their professional roles improves patients' outcomes. Barriers were similar to pharmacists in other countries. Publication of pharmacists' practice research results is important to expand their roles. Collaboration between faculties and pharmacists is a challenge for practice research development in the Japanese community setting.
\end{abstract}

\section{Keywords}

Pharmacy Practice Research, Barrier, Community Pharmacist in Japan

\footnotetext{
"Corresponding author.
}

How to cite this paper: Sawada, Y., Takehira, R. and Yamamura, S. (2015) Japanese Community Pharmacists' Barriers to Conducting or Participating in Practice Research. Pharmacology \& Pharmacy, 6, 421-427. 


\section{Introduction}

In the joint International Pharmaceutical Federation/World Health Organization guidelines on good pharmacy practice, there are four main roles where society and the individuals served expect pharmacists' involvement or supervision. They should:

1) Prepare, obtain, store, secure, distribute, administer, dispense, and dispose of medical products;

2) Provide effective medication therapy management;

3) Maintain and improve professional performance;

4) Contribute to improve effectiveness of the healthcare system and public health [1].

These pharmacists' roles are patient-focused rather than product-oriented. Community pharmacists who are health professionals close to community people should expand their patient-focused profession. To do so, they should have more confidence in the fact that the pharmacists' pharmacy practice research improves patients' outcomes. Pharmacy practice research is a type of health service research that focuses on pharmacist care and its effect on patient outcomes [2].

There are reports that pharmacists' involvement in pharmacy practice research will increase patient outcomes [3]-[5]. However, it has been reported that there are some barriers for pharmacists to engage in practice research [6]-[8].

In Japan, there are three major annual conferences that many community pharmacists attend: annual meetings of The Pharmaceutical Society of Japan (PSJ) [9], Japanese Society of Pharmaceutical Health Care and Sciences (JSPHCS) [10], and Japan Pharmaceutical Association (JPA) [11]. Although there are many posters or oral conference presentations by community pharmacists, few publish reports and papers. This suggests that practice research by community pharmacists in Japan has not been very active. Japanese community pharmacists had some barriers to conducting practice research. In this report, we tried to identify Japanese community pharmacists' barriers to conducting research.

\section{Method}

Community pharmacists who presented at three major pharmacy-related conferences in 2012 and 2013 were selected from the annual meetings' abstracts. Their addresses were identified from the author's occupation. Community pharmacists $(n=478)$ were selected in this research.

After obtaining informed consent to contribute in this survey, they were asked about the difficulties in presentations and necessary assistance for improving them. They were also asked about barriers to conducting practice research in their practical settings. A questionnaire was mailed to pharmacists directly and responses were collected by a postcard without their name and address. There is no personal identifier in responses (Appendix). Because that all responses were answered their year of practical experience (missing 4), we assessed that responses were from pharmacists themselves we mailed.

The questionnaire included age, gender, years of experience in the present workplace, presentation content, difficult points in the presentation, necessary assistance for improving the presentation, and barriers to conducting practice research. The research and design were approved by the OTC Self-Medication Promotion Foundation.

Data handling of collected data was carried out by JMP 11.0 (SAS Institute Japan, Tokyo).

\section{Results}

Of the 478 pharmacists who were mailed the questionnaire, 230 (47.9\%) completed the survey. Table 1 summarizes the community pharmacist characteristics. Many pharmacists in their 30s or 40s who had 5 - 20 years practice experience responded. It took five years of practical experience to conduct practice research. Most pharmacists presented at the annual JPA meeting because it is an association mainly organized by community pharmacists. The mean number of past presentation experiences were 2.3 and the inter quartile range was $1-3$. More than one-thirds was presentations introducing their daily practice. Many of them provided little evidence in their pharmacy practice research. Intervention studies, a higher evidence level design, comprised only $8 \%$ of all presentations. About $60 \%$ of presentations were presented by poster.

Table 2 shows their difficulties when presenting and support necessary for presentation preparation. They had difficulty organizing and discussing their presentations and lacked time to do in-depth research. Funds for 
Table 1. Characteristics of community pharmacists.

\begin{tabular}{|c|c|}
\hline Characteristics & Total $n=230$ \\
\hline \multicolumn{2}{|l|}{ Gender, $\mathrm{n}(\%)$ Missing 3} \\
\hline Male & $152(67.0)$ \\
\hline Female & $75(33.0)$ \\
\hline \multicolumn{2}{|l|}{ Age, $n$ (\%) Missing 1} \\
\hline $20 \mathrm{~s}$ & $27(11.8)$ \\
\hline $30 \mathrm{~s}$ & $93(40.6)$ \\
\hline $40 \mathrm{~s}$ & $54(23.6)$ \\
\hline $50 \mathrm{~s}$ & $39(17.0)$ \\
\hline$>60 \mathrm{~s}$ & $16(7.0)$ \\
\hline \multicolumn{2}{|l|}{ Years of practical experience, $n$ (\%) Missing 4} \\
\hline$<2$ years & $7(3.1)$ \\
\hline 2 - 5 years & $33(14.6)$ \\
\hline 5 - 10 years & $74(32.7)$ \\
\hline 10 - 20 years & $70(31.0)$ \\
\hline$>20$ years & $42(18.6)$ \\
\hline \multicolumn{2}{|l|}{$\begin{array}{l}\text { Congress to present, } \mathrm{n}(\%) \\
\text { (multiple answers allowed) }\end{array}$} \\
\hline $\operatorname{PSJ}^{1)}$ & $56(24.3)$ \\
\hline$J S P H C S^{2)}$ & $50(21.7)$ \\
\hline $\mathrm{JPA}^{3)}$ & $200(87.0)$ \\
\hline $\begin{array}{l}\text { Number of past presentations experienced } \\
\text { mean (range, IQR) }\end{array}$ & $2.3(1-46,1-3)$ \\
\hline \multicolumn{2}{|l|}{ Type of research, n (\%) Missing 7} \\
\hline Introduction of practice & $94(42.1)$ \\
\hline Observational study & $68(30.5)$ \\
\hline Intervention study & $18(8.1)$ \\
\hline Others & $43(19.3)$ \\
\hline \multicolumn{2}{|l|}{ Type of presentation, $n$ (\%) Missing 4} \\
\hline Oral & $91(40.3)$ \\
\hline Poster & 134 (59.3) \\
\hline Others & $1(0.4)$ \\
\hline
\end{tabular}

1) PSJ: The annual congress of The Pharmaceutical Society of Japan [9]; 2) JSPHCS: The annual congress of the Japanese Society of Pharmaceutical Health Care and Sciences [10]; 3) JPA: The annual meeting of the Japan Pharmaceutical Association [11].

\section{Table 2. Difficulties and Necessary Support for Presentations.}

\begin{tabular}{ccc}
\hline Item & Difficulty & Needed Support \\
\hline Organize the presentation & $98(42.6)$ & $81(35.2)$ \\
Lack of time to research & $83(36.1)$ & $70(30.4)$ \\
Discussion in the presentation & $77(33.5)$ & $73(31.7)$ \\
Statistical analysis & $59(25.7)$ & $91(39.6)$ \\
Preparation of abstract & $46(20.0)$ & $49(21.3)$ \\
Shortage of human resources & $34(14.8)$ & $41(17.8)$ \\
Design the study & $26(11.3)$ & $43(18.7)$ \\
Funds & $8(3.5)$ & $15(6.5)$ \\
English expression & $7(3.0)$ & $15(6.5)$ \\
Others & $24(10.4)$ & $19(8.3)$ \\
\hline
\end{tabular}

Numbers (\%) in 230 responses. 
research were not a significant issue for most, because many presentations were developed from their daily practice. The most necessary support was statistical analysis. People with statistical knowledge and skills were not found in their community pharmacy settings.

Table 3 shows community pharmacists' barriers in conducting practice research. The biggest barrier was insufficient research time. Because they were busy in daily practice, they had insufficient time to research. Other major barriers were no practice research supervisors and other community pharmacists' lack of understanding practice research's importance. Other items relating to workplaces and coworkers were also barriers to conducting practice research for Japanese community pharmacists. Shortage of research funds was not a major barrier for practice research.

\section{Discussion}

To expand the patient-focused professional role of Japanese community pharmacists, they should clarify that their professional roles improve patients' outcomes through practice research. It would be important to identify and fix community pharmacists' barriers to conducting practice research.

Participants in this research had strong interest in research because they presented at pharmacist-related meetings. Many of the community pharmacists' presentations were merely introductions of their daily practice, not pharmacy practice research. This result indicates that pharmacy practice research by Japanese community pharmacists has yet to be active.

The biggest barrier to conduct pharmacy practice research was lack of time to research. Simpson et al. reported that time was the greatest barrier to participation of pharmacy practice-based research in Canada [6]. In a systematic review of 15 studies completed in the UK, Australia, and Canada, the barriers were: lack of time and workload; insufficient or lack of funds; and lack of research knowledge, training, mentorship, and support. [3] Armour et al. reported the barriers to research participation were pharmacists' mindsets, communication, infrastructure (time, money, and staff), and skills/knowledge [12]. In Japan, community pharmacists have similar barriers to practice research participation. However, money and funds were not significant barriers in Japan. This was because Japanese community pharmacists did not have the experience to participate in well-designed practice research. Pharmacists' culture may be a barrier for practice research [7] [13]. Pharmacists' culture is strongly dependent on country and region. It is unclear whether the culture of Japanese pharmacists would affect the barriers to practice research participation. Further studies are needed to determine whether pharmacists' culture influences them to participate in practice research.

Other barriers arise from educational issues. Half of them had more than five years of practice experience; this suggests that it is necessary to have five years' practical experience to learn how to organize their experiences for conference presentations. Many pharmacists identify no research supervisor in their practice sites. This was a reason they had difficulties in presentation organization and discussion.

In recent research, research experience prior to residency training strongly predicted subsequent practice research publication [14]. This report noted that practice research training for entry-level pharmacists would be important for them to conduct practice research. Many Japanese community pharmacists did not receive practice

Table 3. Barriers for Implementing Practice Research.

\begin{tabular}{|c|c|c|c|c|}
\hline Item & Major & Small & Less & No \\
\hline Lack of time to research & $124(55.9)$ & $72(32.4)$ & $21(9.5)$ & $5(2.3)$ \\
\hline No supervisor & $61(27.7)$ & $92(41.8)$ & $44(20.0)$ & $23(10.5)$ \\
\hline Lack of support from other pharmacists & $44(20.3)$ & 78 (35.9) & $61(28.1)$ & $34(15.7)$ \\
\hline Lack of support from colleagues except for pharmacists & $30(13.8)$ & $81(37.3)$ & $68(31.3)$ & $38(17.5)$ \\
\hline Lack of understanding practice research importance & $37(17.2)$ & $71(33.0)$ & $66(30.7)$ & $41(19.1)$ \\
\hline Shortage of research funds & $28(12.9)$ & $63(29.0)$ & $81(37.3)$ & 45 (20.7) \\
\hline No understanding by boss & $36(16.7)$ & 45 (20.8) & $71(32.9)$ & $64(29.6)$ \\
\hline Research not pay-related & $24(11.1)$ & $53(24.4)$ & $75(34.6)$ & $65(30.0)$ \\
\hline
\end{tabular}

Numbers (\%) in 230 responses. 
research training when they were in pharmacy school. Possible other reasons would be a lack of understanding of practice research's importance as well as people not knowing what "practice research for evidence" actually is. In Japan, if they learned how to conduct pharmacy practice research in pharmacy school, they could begin practice research earlier.

Establishment of collaboration between faculties and pharmacists would be a challenge for practice research development by Japanese community pharmacists. To lower the barriers, education to community pharmacists on how to implement practice research through continuing professional development (CPD) programs would be essential for practice research development in their practice settings.

\section{Limitations}

The results are based on questionnaire responses by community pharmacists who presented at conferences. They had stronger interest in research than other pharmacists. Other pharmacists would have more barriers to conduct or participate in practice research than participants in this research. Therefore, the actual rate of those experiencing barriers would be much higher than the rates indicated in this report.

\section{Conclusion}

The barriers for Japanese community pharmacists to conduct or participate in pharmacy practice research were lack of time, no supervisor, and other community pharmacists' lack of understanding practice research importance. These points are similar to barriers for pharmacists in other countries, but money and funds were not major barriers for Japanese community pharmacists. Other barriers were educational issues in pharmacy school and CPD. Publishing practice research outcomes by community pharmacists would be important to expand pharmacists' roles in the society. To overcome the barriers, collaboration between faculties and community pharmacists would necessary and be a challenge. If Japanese community pharmacists participate in practice research and make evidence themselves, the pharmacists will be able to show evidently that they can contribute to improve Japanese public health.

\section{Acknowledgements}

We acknowledge to Ms. R. Hirokawa, A. Hirano and M. Fujii for their technical assistance.

\section{Conflict of Interest}

This research was supported by OTC Self-Medication Promotion Foundation.

\section{References}

[1] World Health Organization (2011) Joint FIP/WHO Guidelines on Good Pharmacy Practice: Standards for Quality of Pharmacy Services. WHO Technical Report Series, No. 961.

http://apps.who.int/medicinedocs/documents/s18676en/s18676en.pdf

[2] Tsuyuki, R.T. (2014) Designing Pharmacy Practice Research Trials. The Canadian Journal of Hospital Pharmacy, 67, 226-229. http://dx.doi.org/10.4212/cjhp.v67i3.1360

[3] Awaisu, A. and Alsalimy, N. (2014) Pharmacists' Involvement in and Attitudes toward Pharmacy Practice Research: A Systematic Review of the Literature. Research in Social and Administrative Pharmacy. (In Press) http://dx.doi.org/10.1016/j.sapharm.2014.12.008

[4] McLean, D.L., McAlister, F.A., Johnson, J.A., King, K.M., Makowsky, M.J., Jones, C.A., et al. (2008) A Randomized Trial of the Effect of Community Pharmacist and Nurse Care on Improving Blood Pressure Management in Patients with Diabetes Mellitus: Study of Cardiovascular Risk Intervention by Pharmacists-Hypertension (SCRIP-HTN). Archives of Internal Medicine, 168, 2355-2361. http://dx.doi.org/10.1001/archinte.168.21.2355

[5] Kraemer, D.F., Kradjan, W.A., Bianco, T.M. and Low, J.A. (2012) A Randomized Study to Assess the Impact of Pharmacist Counseling of Employer-Based Health Plan Beneficiaries with Diabetes: The EMPOWER Study. Journal of Pharmacy Practice, 25, 169-179. http://dx.doi.org/10.1177/0897190011418513

[6] Simpson, S.H., Johnson, J.A., Biggs, C., Biggs, R.S., Kuntz, A., Semchuk, W., et al. (2001) Practice-Based Research: Lessons from Community Pharmacist Participants. Pharmacotherapy, 21, 731-739. http://dx.doi.org/10.1592/phco.21.7.731.34570 
[7] Rosenthal, M.M., Breault, R.R., Austin, Z. and Tsuyuki, R.T. (2003) Pharmacists' Self-Perception of Their Professional Role: Insights into Community Pharmacy Culture. Journal of American Pharmacists Association, 51, 363-367.

[8] Hébert, J., Laliberté, M.C., Berbiche, D., Martin, E. and Lalonde, L. (2013) The Willingness of Community Pharmacists to Participate in a Practice-Based Research Network. Canadian Pharmacists Journal, 146, 47-54. http://dx.doi.org/10.1177/1715163512473240

[9] The Pharmaceutical Society of Japan. http://www.pharm.or.jp/index_e.html

[10] Japanese Society of Pharmaceutical Health Care and Sciences. (In Japanese) http://www.jsphcs.jp/index.php

[11] Japan Pharmaceutical Association. http://www.nichiyaku.or.jp/e/default.html

[12] Armour, C., Brillant, M. and Krass, I. (2007) Pharmacists' Views on Involvement in Pharmacy Practice Research: Strategies for Facilitating Participation. Pharmacy Practice, 5, 59-66. http://dx.doi.org/10.4321/s1886-36552007000200002

[13] Scahill, S., Harrison, J., Carswell, P. and Babar, Z.U. (2009) Organisational Culture: An Important Concept for Pharmacy Practice Research. Pharmacy World \& Science, 31, 517-521. http://dx.doi.org/10.1007/s11096-009-9318-8

[14] Pinelli, N.R., Sikora, A.N., Witherspoon, L.A., Rao, K.V. and Rhoney, D.H. (2015) Impact of Pharmacy Residency Research Training on Residents’ Actual versus Perceived Ability and Interest to Identify and Solve Practice-Related Problems. Journal of Pharmacy Practice. (In Press) 


\section{Questionnaire (In Part)}

Please answer questions by the number then reply your response by attached postcard

Please NOT write your name and address in a postcard

1. Age
1) $20 \mathrm{~s}$
2) $30 \mathrm{~s}$
3) $40 \mathrm{~s}$
4) $50 \mathrm{~s}$
5) $>60 \mathrm{~s}$

2. Gender

1) Female, 2) Male

3. Years of practical experience
1) $<2$ years
2) 2 - 5 years
3) 5 - 10 years
4) 10 - 20 years
5) $>20$ years

4. Congress to present (multiple answers allowed)

1) The annual congress of The Pharmaceutical Society of Japan

2) The annual congress of the Japanese Society of Pharmaceutical Health Care and Science

3) The annual meeting of the Japan Pharmaceutical Association

5. Type of research

1) Introduction of practice, 2) Observational study, 3) Intervention study, 4) Others

6. Number of past presentation experienced: ( ) times

7. Type of presentation
1) Oral
2) Poster
3) Others

8. Points of Difficult for Presentations (multiple answers allowed)
1) Preparation of abstract
2) Organize presentation
3) Select study design
4) Statistical analysis
5) Discussion in the presentation
6) Preparation of poster/slides
7) Shortage of funds
8) Lack of Human resources

9) English expression in poster/slides/abstract
10) Lack of time to research
11) Others

9. Necessary Support for Presentations (multiple answers allowed)
1) Correction of abstract
2) Advice to organize presentation
3) Advice to select study design
4) Advice of statistical analysis
5) Support for discussion in the presentation
6) Advice of preparation of poster/slides
7) Financial support
8) Support of Human resources
9) Advice for English expression in poster/slides/abstract
10) Time to research
11) Others

10. Barriers for Implementing of Practice Research (1. Major, 2. Small
a) Shortage of research fund
b) Lack of time to research
c) No supervisor
d) No understanding by boss
e) Lack of support from other pharmacists
f) Lack of support from colleagues except for pharmacists
g) Research won't be a pay-related
h) Lack of understanding of importance of practice research
i) Others 